\title{
Les nouvelles directives de l'ASSM «Soins palliatifs»
}

Reconnaissant l'importance des soins palliatifs en médecine l'Académie Suisse des Sciences Médicales (ASSM), à l'initiative de sa Commission Centrale d'Ethique (CCE), a mis à son programme le soutien financier de projets de recherche et d'enseignement dans le domaine de l'approche palliative. Qui plus est, la CCE a reconnu le besoin d'insister sur le rôle central des soins palliatifs dans plusieurs des directives médico-éthiques récemment élaborées et publiées. Ce faisant, la CCE a pris conscience de la nécessité de rédiger des directives médico-éthiques spécifiques à ce domaine afin de clarifier sa définition, ses concepts, sa place dans l'approche thérapeutique, ses domaines d'application et ses relations avec la médecine curative.

L'empathie peut être mise à la base des soins palliatifs. Ce sens de l'empathie est avant tout une affaire d'éducation, mais il doit être approfondi et affiné au cours des études, renforcé par des considérations éthiques et étayé par une vue humaniste de la relation patient-corps médical, et constamment revivifié durant les formations postgrades et continues. Et cela, quelle que soit la spécialité à laquelle les médecins, les divers thérapeutes et les membres de l'équipe de soins infirmiers se sont destinés.

Un autre point important de ces directives réside dans la reconnaissance mutuelle des médecins et des partenaires des soins palliatifs lorsqu'il doit être fait appel à l'un d'eux ou plusieurs d'entre eux. Une collaboration étroite pour un plan de traitement, établi en consensus, et auquel le patient comme ses proches bien informés adhèrent, sera mise en place et ce plan régulièrement réactualisé en fonction de l'évolution du patient.

Dans cette perspective, le rôle central d'une information précise et d'une communication ouverte et sincère est soulignée. Les traitements à visée curative et les soins palliatifs ne doivent pas évoluer en parallèle, mais se fondre en un tout harmonieux. Les traitements dits symptomatiques appartiennent aux deux domaines sans qu'il y ait césure. Au fur et à mesure que l'approche curative atteint ses limites et que celle des soins palliatifs gagne en importance, c'est de moins en moins la maladie et de plus en plus le patient et sa qualité de vie qui doivent être au centre des préoccupations et de l'attention générale.

Finalement, afin d'évoluer vers toujours plus d'efficacité, les soins palliatifs doivent aussi se soumettre à l'auto-évaluation par de régulières discussions de cas et des projets de recherche réunissant les spécialités concernées.

Nous invitons non seulement les médecins, mais aussi les autres soignants et acteurs engagés dans les soins palliatifs, à prendre connaissance sur notre site (www.asm.ch) de ce projet de directives médico-éthiques et à nous faire part de leurs réactions.

Prof. Michel B. Vallotton, président CCE Prof. Peter Suter, président ASSM 


\title{
Soins palliatifs: prise en charge complète des patients atteints de maladies incurables ou chroniques et évolutives ou qui mettent la vie gravement en danger
}

\author{
L'ASSM publie I'ébauche de nouvelles directives
}

\author{
R. Kunz ${ }^{a}$, M. Salathéb
}

La promotion et le soutien des soins palliatifs figurent depuis longtemps déjà à l'ordre du jour. Le développement de l'offre palliative a été tout particulièrement réclamé lors du débat sur l'assistance au suicide ou l'euthanasie active*. La valeur de l'approche palliative reste cependant controversée et son ancrage sur les plans national et cantonal insuffisant, d'autant plus que l'interprétation et la compréhension de la notion de soins palliatifs sont variables. L'Académie Suisse des Sciences Médicales (ASSM) a engagé une réflexion sur ce sujet et a adopté fin novembre des directives médico-éthiques et recommandations pour la mise en consultation; leur but est d'encourager l'introduction des soins palliatifs dans la pratique médicale quotidienne.

a Président de la sous-commission «Soins palliatifs»

b Collaboratrice scientifique ASSM

\footnotetext{
* voir p.ex. la prise de position du Conseil fédéral du 14 novembre 2001 face à la motion G. Zäch «Euthanasie. Combler les lacunes de la loi au lieu d'autoriser l'homicide». Le Conseil fédéral a souligné à quel point il est important d'exploiter les possibilités offertes par la médecine et les soins palliatifs, et a chargé le DFJP et le DFI de promouvoir l'approche palliative dans le cadre des réformes touchant à la santé publique.
}

Correspondance:

lic. iur. Michelle Salathé

Académie Suisse

des Sciences Médicales

Petersplatz 13

CH-4051 Bâle

E-mail: m.salathe@samw.ch
Il est question d'approche palliative dans diverses directives médico-éthiques publiées ces dernières années par l'ASSM. Les définitions sont hétérogènes et l'importance accordée aux soins palliatifs au sein de l'activité médicale reste controversée. C'est pourquoi la Commission Centrale d'Ethique a chargé une sous-commission d'élaborer des directives sur ce thème.

Les nouvelles directives entendent définir les soins palliatifs de manière complète sur la base d'une conception bio-psycho-sociale de la maladie, non seulement en fin de vie, mais pendant toute la durée d'une maladie évolutive: la qualité de vie n'est pas en premier lieu une notion médicale, elle se définit au contraire dans le contexte biographique; c'est le point de vue du patient qui est déterminant. Lors d'un traitement et d'une prise en charge, il faut considérer les aspects psychiques, sociaux et spirituels conformément aux souhaits du patient - et non pas exclusivement les symptômes physiques.

Ces directives s'adressent aux collaborateurs impliqués dans la prise en charge de personnes gravement malades dans les domaines ambulatoire et hospitalier: à savoir médecins, infirmières et spécialistes de différentes disciplines, allant de la néonatologie à la gériatrie et la psychiatrie, en passant par les soins intensifs. En médecine, un traitement curatif et une approche palliative ne peuvent bien souvent pas être clairement distingués et finissent par se compléter. De nombreuses maladies, comme des maladies cardiaques, pulmonaires et rénales chroniques, mais également des maladies neurologiques, peuvent induire, en plus d'une invalidation et d'une dépendance croissantes, des situations dans lesquelles le pronostic vital est menacé. Dans ces cas et parallèlement au soulagement des symptômes, les soins palliatifs comprennent aussi une aide à surmonter la maladie et à prendre en compte de possibles aggravations. Le but de ces soins est de maintenir la meilleure qualité de vie possible au patient jusqu'à son décès.

Les directives ont pour but d'encourager le personnel soignant à adopter une attitude qui va reconnaître les limites de la médecine et faire face à la situation du patient et au sentiment d'impuissance qui transparaît souvent dans pareils cas. L'attitude fondamentale à adopter par l'équipe soignante sera abordée à divers endroits dans les directives. L'observation de valeurs centrales, telles que le respect de la dignité du patient et son autonomie, ainsi qu'une communication franche et appropriée sont mises en évidence, tandis qu'est décrite la manière de les appliquer au quotidien dans le milieu médical. L'attitude adoptée par le personnel soignant n'a

Fin novembre 2005, le Sénat a adopté les directives «Soins palliatifs» pour la mise en consultation; les directives peuvent être consultées sur internet à l'adresse www. samw.ch ou commandées au Secrétariat général de l'ASSM. Le délai de consultation s'achève fin février 2006; les prises de position sont à adresser au Secrétariat général de l’ASSM, Petersplatz 13, 4051 Bâle. 
pas seulement son importance face au patient et à ses proches, mais également dans les rapports entre accompagnants. La continuité dans le traitement et la prise en charge ainsi que le travail en réseau interdisciplinaire constituent des conditions-cadres essentielles à l'approche palliative. L'acceptation et l'estime mutuelles ainsi que la connaissance et le respect de ses propres limites et possibilités sont indispensables à une collaboration fructueuse.

Les directives abordent également les malentendus, dangers et attentes déraisonnables dont les soins palliatifs font l'objet. A titre d'exemple, on peut citer l'attente de voir tous les symptômes lourds être soulagés à tout moment, ou la diminution systématique des demandes de suicide assisté ou d'euthanasie active, lorsque les propositions de soins palliatifs sont suffisantes. L'opinion répandue qui veut que tous les traitements qui soulagent soient des soins palliatifs, ou encore celle qui fait correspondre l'approche palliative au renoncement à des traitements curatifs, est également erronée.

Les recommandations qui s'adressent en particulier aux institutions du système de la santé, de la formation prégraduée, postgraduée et continue, ainsi qu'aux autorités politiques et aux bailleurs de fonds vont également dans ce sens. Outre la promotion, le soutien et l'ancrage des soins palliatifs dans les programmes de formation, c'est aussi la révision des incitations tarifaires qui est stimulée. Le mode actuel de financement empêche en partie d'évoluer vers une approche palliative: les thérapies déjà engagées continuent généralement à être financées complètement en hôpital de soins aigus, le renoncement à une thérapie ou la bifurcation vers des options palliatives conduisant au contraire rapidement à entrer dans le domaine des soins à long terme, ce qui représente des charges finan- cières considérables pour le patient et sa famille. D'autre part, le risque de prodiguer des soins palliatifs à la place de mesures thérapeutiques raisonnables et d'options thérapeutiques, pour des raisons économiques, existe toujours.

Il est indiscutable que les présentes directives sont très exigeantes avec le personnel médical concerné et les institutions. C'est pourquoi les questions d'ordre éthique se posent avant tout par rapport au tracé des frontières. Dans le traitement et l'accompagnement de personnes atteintes de maladies incurables ou mourantes, il convient de définir des limites individuelles qui ne sont pas uniquement guidées par des impératifs techniques et des directives professionnelles, mais qui sont également orientées vers la situation personnelle du patient. La finalité des soins palliatifs est de renforcer la qualité de vie, indépendamment des limites inhérentes aux moyens curatifs.

Membres de la sous-commission responsable de l'élaboration des directives

Dr méd. Roland Kunz, Schlieren, président; Dr méd. Urs Aemissegger, Winterthur; Dr méd. Eva Bergsträsser, Zurich; Dr méd. Christian Hess, Affoltern am Albis; Prof. Dr méd. Christoph Hürny, Saint-Gall; Mme Christine Kaderli, Nussbaumen, infirmière; Dr méd. Hans Neuenschwander, Lugano; Mme Françoise Porchet, Lausanne, infirmière; Mme Susan Porchet, Birchwil, musico-thérapeute; PD Dr méd. Bara Ricou, Genève; lic. iur. Michelle Salathé, Bâle (ex officio); Mme Nelly Simmen, Berne, infirmière; Dr théol. Plasch Spescha, Berne, aumônier et éthicien; Prof. Dr méd. Michel B. Vallotton, Genève, Président de la CCE (ex officio) 